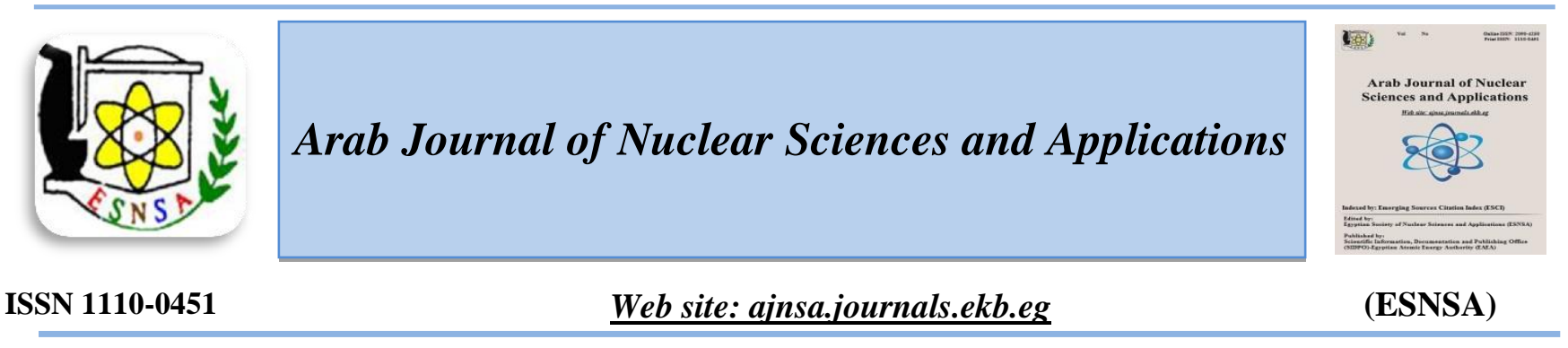

\title{
A Uniform Laser Pulse for a Solid Cylinder Using Different Theories of Magneto Thermoelasticity
}

\author{
S.E. Khader ${ }^{1}$, Mona El. M. Khedr ${ }^{2}$ \\ ${ }^{1}$ Mathematical and Theoretical Physics Department, Nuclear Research Center, Atomic Energy Authority, Egypt \\ ${ }^{2}$ Physics Department, Faculty of Science University of Alexandria, EGYPT
}

\begin{abstract}
Received $21^{\text {st }}$ Feb. 2018 This work is concerned with the study of vibrations induced by a laser beam in the context of different Accepted $7^{\text {th }}$ Dec. 2018 theories of magneto thermoelasticity, coupled thermoelasticity (CTE), thermoelasticity with one relaxation time (L-S) and thermoelasticity without energy dissipation (G-L), of an infinitely long solid conducting circular cylinder. The surface of the cylinder is assumed to be traction free. The temporal profile of the laser beam is considered as non-Gaussian. The cylinder is considered to be made of an isotropic homogeneous thermoelastic material put in a uniform magnetic field in the direction of the axis. This produces an induced magnetic field and an induced electric field. Laplace transform techniques are used to derive the solution in the Laplace transform domain. The inversion process is carried out using a numerical method based on Fourier series expansions. The temperature, displacement, stresses, induced magnetic field and induced electric field are calculated numerically, and graphically represented.
\end{abstract}

Keywords: Magneto thermoelasticity; Coupled thermoelasticity; Generalized thermoelasticity; Non-Gaussian laser pulse

\section{Introduction}

The dynamic interactions between the thermal and mechanical fields in solids are important due to their numerous applications in the field of geophysics, plasma physics and related topics, especially in the nuclear field and high-speed particle accelerators. The theory of generalized thermoelasticity with one relaxation time was introduced by Lord and Shulman [1]. In this theory Cattaneo -Maxwell law of heat conduction replaces the conventional Fourier's law. The heat equation associated with this theory is a hyperbolic one and hence it automatically eliminates the paradox of infinite speeds of propagation inherent in both the uncoupled and the coupled theories of thermoelasticity. For many problems involving steep heat gradients and when short time effects are sought, this theory is indispensable. Sherief and El-Maghraby proposed a solution to some crack problems related to this theory [2-3]. Sherief and Hamza have successfully presented the solution of axisymmetric problems in spherical regions [4] and in cylindrical regions [5]. Sherief and Ezzat have offered the solution in the form of series [6]. Sherief and Dhaliwal used asymptotic expansions to obtain the solution of a 1D problem and to find the locations of the wave fronts and the speed of propagation of thermoelastic waves [7].

Corresponding author: shabolaaaa@yahoo.com

DOI: 10.21608/ajnsa.2018.2043.1062

(C) Scientific Information, Documentation and Publishing Office (SIDPO)-EAEA 
This theory was extended to deal with micropolarity of the medium [8], viscoelastic effects [9]. This subject has been studied by other workers [10-12]. An increasing attention is being devoted to the interaction between magnetic fields and strain in a thermoelastic solid due to its numerus applications. In these investigations, the heat equation under consideration is usually taken as the uncoupled or the coupled equation and not the generalized one. This attitude is justified in some situations where the solutions obtained, using any of these equations, differ quantitatively to a small extent. However, when short time effects are considered, the full, generalized system of equations has to be used, otherwise a great deal of accuracy is lost [1].

Among the authors who considered the generalized magneto-thermoelastic equations are Nayfeh and Nemat-Nasser [13] who studied the propagation of plane waves in a solid under the influence of an electromagnetic field. These authors have obtained the governing equations in the general case and the solution for some particular cases. Sherief and Khader [14] studied Propagation of discontinuities in electromagneto generalized thermoelasticity in cylindrical regions and calculated the speed of waves.

Green and Lindsay [15] developed the theory of generalized thermoelasticity with two relaxation times, based on a generalized inequality of thermodynamics. In this theory both the equations of motion and of heat conduction are hyperbolic. The heat conduction law is the same as Fourier's law when the system has a centre of symmetry. Among the contributions to this theory are the works in [16-17].

Green and Nagdhi [18-20] have formulated a new model of thermoelasticity. This model predicts that the internal rate of production of entropy is identically zero, i.e., there is no dissipation of thermal energy. This is known as thermoelasticity without energy dissipation theory (GN theory). In the development of this theory, the thermal displacement gradient is considered as a constitutive variable, whereas in the conventional development of a thermoelasticity theory, the temperature gradient is taken as a constitutive variable [12]. A couple of uniqueness theorems have been proved [21-22], and one-dimensional waves in a half-space and in an unbounded body have been studied [23-25].

\section{Basic equations}

Let $(r, \varphi, z)$ be cylindrical polar coordinates with the $\mathrm{z}$-axis coinciding with the axis of a solid infinitely long elastic circular cylinder of a homogenous, isotropic material of radius a having finite conductivity at a uniform temperature $T_{0}$. The surface of the cylinder is assumed to be traction free. A constant magnetic field of strength $H_{0}$ acts in the direction of the z-axis. This produces an induced magnetic field $h$ and an induced electric field E. Because of the cylindrical symmetry of the problem, all the electro-magnetic quantities satisfy Maxwell's equations.

$$
\operatorname{curl} \underline{\mathbf{h}}=\underline{\mathbf{J}}+\varepsilon_{0} \frac{\partial \underline{\mathbf{E}}}{\partial \mathrm{t}}
$$

$\operatorname{curl} \underline{\mathbf{E}}=-\mu_{0} \frac{\partial \underline{\mathbf{h}}}{\partial t}$,

$\operatorname{div} \underline{\mathbf{h}}=0 \quad, \quad \operatorname{div} \underline{\mathbf{E}}=0$,

$$
\underline{\mathbf{B}}=\mu_{0}\left(\underline{\mathbf{H}}_{\mathbf{0}}+\underline{\mathbf{h}}\right) \quad, \quad \underline{\mathbf{D}}=\varepsilon_{0} \underline{\mathbf{E}}
$$

where $J$ is the electric current density. $\varepsilon_{0}$ and $\mu_{0}$ are the electric and magnetic permeability's, respectively and $\mathrm{B}, \mathrm{D}$ are the magnetic and electric induction vectors, respectively.

Ohm's law for moving media states that $\underline{\mathbf{J}}=\sigma_{o}\left(\underline{\mathbf{E}}+\mu_{0} \frac{\partial \underline{\mathbf{u}}}{\partial t} \times\left(\underline{\mathbf{H}}_{\mathbf{0}}+\underline{\mathbf{h}}\right)\right)$,

where $\sigma 0$ is the electric conductivity and $u$ is the displacement vector. This equation can be literalized by neglecting small quantities of the second order giving

$$
\underline{\mathbf{J}}=\sigma_{o}\left(\underline{\mathbf{E}}+\mu_{0} \frac{\partial \underline{\mathbf{u}}}{\partial t} \times \underline{\mathbf{H}}_{\mathbf{0}}\right) .
$$

The basic equations represented by (CTE), (L-S) and (G-L) can be formulated in the following unified system: The equations of motion have the form

$$
\mu u_{i, j j}+(\lambda+\mu) u_{j, j i}+F_{i}-\gamma\left(1+\tau_{1} \frac{\partial}{\partial t}\right) \mathrm{T}_{, i}=\rho \ddot{u}
$$

The equation of heat conduction has the form 
$k T_{, i i}=\rho c_{E}\left(1+\tau_{2} \frac{\partial}{\partial t}\right) \frac{\partial T}{\partial t}+\gamma T_{0}\left(1+n \tau_{2} \frac{\partial}{\partial t}\right) \dot{u}_{j, j}-Q=\sigma_{o}\left(E-\mu_{0} H_{0} \frac{\partial u}{\partial t}\right)$

The components of the stress tensor oij are given by

$$
\sigma_{i j}=\mu\left(u_{i, j}+u_{j, i}\right)+\left[\lambda u_{i, i}-\gamma\left(1+\tau_{1} \frac{\partial}{\partial t}\right) T\right] \partial_{i, j}
$$

Where $\lambda$ and $\mu$ are Lamé's modulii, $T$ is the absolute temperature of the medium, and $\gamma$ is a material constant given by $\gamma=(3 \lambda+2 \mu) \alpha_{t}$ where $\alpha_{t}$ is the coefficient of linear thermal expansion, $\mathrm{T} 0$ is a reference temperature assumed to be such that $\mid($ $\left.T-T_{0}\right) / T_{0} \mid<<1 . k$ is the thermal conductivity of the medium, $c_{E}$ is the specific heat at constant strain, $\tau_{1}, \tau_{2}$ are the relaxation times and $Q$ the external heat flux. $\rho$ is the density and $F$ is the Lorentz force given by

$$
\begin{aligned}
& \underline{\mathbf{F}}=\underline{\mathbf{J}} \times \underline{\mathbf{B}} \\
& F_{r}=J \mu_{0}\left(H_{0}+h\right), F_{\phi}=F_{z}=0 .
\end{aligned}
$$

From equations (6)-(8)

1- At $\tau_{1}=\tau_{2}=0$ the equations, reduce to coupled thermoelasticity (CTE).

2- At $n=1, \tau_{1}=0, \tau_{2}>0$, the equations reduce to Lord-Shulman (L-S) model.

3- At $n=0, \tau_{1}>0, \tau_{2}>0$, the equations reduce to Green-Lindsay (G-L) model.

The applied magnetic field $\mathrm{H} 0$ has components

$$
H_{0}=\left(0,0, H_{0}\right) \text {. }
$$

We assume that the induced magnetic field has the components

$$
h=(0,0, h)
$$

Eliminating $\mathbf{J}$ between equations (9) and (11), we obtain

$$
\frac{\partial h}{\partial r}=\sigma_{0} \mu_{0} H_{0} \frac{\partial u}{\partial t}-\left(\sigma_{0} E+\varepsilon_{0} \frac{\partial E}{\partial t}\right)
$$

Eliminating E between equations (10) and (12), we get

$$
\left[\nabla^{2}-\sigma_{0} \mu_{0} \frac{\partial}{\partial t}-\mu_{0} \varepsilon_{0} \frac{\partial^{2}}{\partial t^{2}}\right] h=\sigma_{0} \mu_{0} H_{0} \frac{\partial e}{\partial t}
$$

where $\nabla^{2}$ is Laplace's operator given by

$$
\nabla^{2}=\frac{\partial^{2}}{\partial r^{2}}+\frac{1}{r} \frac{\partial}{\partial r}
$$

The displacement $\mathrm{u}$ has components

$$
u=(u(r, t), 0,0) \text {. }
$$

This displacement field will produce the following strain components

$\mathrm{e}_{\mathrm{rr}}=\frac{\partial \mathrm{u}}{\partial \mathrm{r}}, \quad \mathrm{e}_{\phi \phi}=\frac{\mathrm{u}}{\mathrm{r}}, \quad \mathrm{e}_{\mathrm{zz}}=\mathrm{e}_{\mathrm{rz}}=\mathrm{e}_{\mathrm{z} \phi}=\mathrm{e}_{\mathrm{r} \phi}=0$.

The cubic dilatation e is thus given by

$e=\frac{\partial u}{\partial r}+\frac{u}{r}=\frac{1}{r} \frac{\partial(r u)}{\partial r}$.

Applying the div operator to both sides of equation (6), the following was obtained:

$(\lambda+2 \mu) \nabla^{2} e+\left[\mu_{0}^{2} \varepsilon_{0} H_{0} \frac{\partial^{2}}{\partial t^{2}}-\mu_{0} H_{0} \nabla^{2}\right] h-\gamma\left(1+\tau_{1} \frac{\partial}{\partial t}\right) \nabla^{2} T=\rho \frac{\partial^{2} e}{\partial t^{2}}$

$\mathrm{E}$ and $\mathrm{J}$ have the components

$$
E=(0, E, 0) \quad \text { and } \quad J=(0, J, 0)
$$

The equation of heat conduction reduces the form

$$
k \nabla^{2} T=\rho c_{E}\left(1+\tau_{2} \frac{\partial}{\partial t}\right) \frac{\partial T}{\partial t}+\gamma T_{0}\left(1+n \tau_{2} \frac{\partial}{\partial t}\right) \frac{\partial e}{\partial t}-Q
$$

$\frac{1}{r} \frac{\partial}{\partial r}(r E)=-\mu_{0} \frac{\partial h}{\partial t}$, 
$\sigma_{r r}=2 \mu \frac{\partial u}{\partial r}+\lambda e-\gamma\left(1+\tau_{1} \frac{\partial}{\partial t}\right) T$

Let the medium is heated uniformly by a laser pulse with non-Gaussian form temporal profile $[18]$ as

$$
L(t)=\frac{L_{0} t}{t_{p}^{2}} e^{-t / t_{p}}
$$

Where tp is a characteristic time (measured by picoseconds) of the laser-pulse (the time duration of a laser pulse), L0 is the laser intensity which is defined as the total energy carried by a laser pulse per unit area of the laser beam. The conduction heat transfer in the medium can be modeled as one-dimensional problem with an energy source $\mathrm{Q}(\mathrm{r}, \mathrm{t})$ near the surface, i.e.

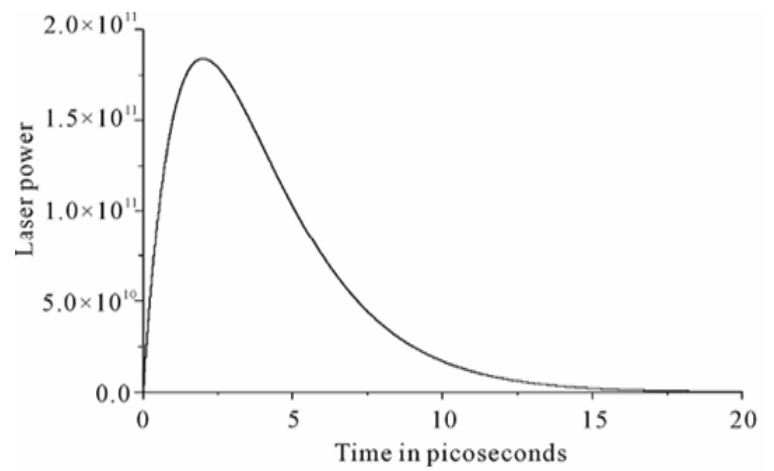

Figure (1) Temporal of laser power $\mathrm{L} / \mathrm{L}_{0}$

$$
\begin{aligned}
Q(r, t) & =\frac{1-R}{\delta_{1}} e^{(r-h / 2) / \delta_{1}}, \\
L(t) & =\frac{R_{a} L_{O}}{\delta_{1} t_{p}^{2}} t e^{(r-h / 2) / \delta_{1}-t / t_{p}}
\end{aligned}
$$

Where $\delta 1$ is the absorption depth of heating energy and $\mathrm{Ra}$ is the surface reflectivity [10]. Note that the laser pulse may lie on the surface of the medium (r $=0$ ) see figure 1 . In this case, the energy source takes the form

$$
Q(r, t)=\frac{R_{a} L_{0}}{\delta_{1} t_{p}^{2}} t e^{h / 2 \delta_{1}-t / t_{p}}
$$

\section{Solution the problem}

Let us introduce the following non-dimension variables

$$
\begin{aligned}
& r^{*}=c_{0} \eta r \quad, \quad u^{*}=c_{0} \eta u, \sigma^{*}{ }_{i j}=\frac{\sigma_{i j}}{\mu}, \\
& \theta=\frac{\gamma\left(T-T_{0}\right)}{\lambda+2 \mu}, \tau_{2}=c_{0}^{2} \eta \tau_{2} \\
& t^{*}=c_{0}^{2} \eta t, \tau_{1}=c_{0}^{2} \eta \tau_{1}, E^{*}=\frac{\eta}{\sigma_{0} \mu_{0}^{2} H_{0} c_{0}} E, \\
& h^{*}=\frac{\eta}{\sigma_{0} \mu_{0} H_{0}} h, \\
& \eta=\frac{\rho c_{E}}{k}, \quad c_{0}^{2}=\frac{\lambda+2 \mu}{\rho} . \\
& Q^{*}=\frac{\gamma Q}{K c_{0}^{2} \eta^{2}(\lambda+2 \mu)}
\end{aligned}
$$

The governing equations (10), (13), (16), (17) and (18) in non-dimensional form become (dropping the asterisks for convenience)

$$
\begin{aligned}
& \frac{1}{r} \frac{\partial}{\partial r}(r E)=-\frac{\partial h}{\partial t} \\
& {\left[\nabla^{2}-v \frac{\partial}{\partial \mathrm{t}}-\mathrm{V}^{2} \frac{\partial^{2}}{\partial \mathrm{t}^{2}}\right] \mathrm{h}=\frac{\partial \mathrm{e}}{\partial \mathrm{t}}}
\end{aligned}
$$

$$
\nabla^{2} e+\varepsilon_{2} v\left[V^{2} \frac{\partial^{2}}{\partial t^{2}}-\nabla^{2}\right] h-\left(1+\tau_{1} \frac{\partial}{\partial t}\right) \nabla^{2} \theta=\frac{\partial^{2} e}{\partial t^{2}}
$$

$$
\nabla^{2} \theta=\left(1+\tau_{2} \frac{\partial}{\partial t}\right) \frac{\partial \theta}{\partial t}+\varepsilon_{1}\left(1+n \tau_{2} \frac{\partial}{\partial t}\right) \frac{\partial e}{\partial t}-Q
$$

$$
\begin{gathered}
\sigma_{r r}=2 \frac{\partial u}{\partial r}+\left(\beta^{2}-2\right) e-\beta^{2}\left(1+\tau_{1} \frac{\partial}{\partial t}\right) \theta \\
v=\frac{\sigma_{0} \mu_{0}}{\eta} \quad, \quad \varepsilon_{1}=\frac{T_{0} \gamma^{2}}{c_{E} \rho^{2} c_{0}^{2}},
\end{gathered}
$$

where

$$
\begin{gathered}
\varepsilon_{2}=\frac{\mu_{0} H_{0}^{2}}{\lambda+2 \mu}, \\
V=\frac{c_{0}}{c}, \quad c^{2}=\frac{1}{\varepsilon_{0} \mu_{0}}, \\
\beta^{2}=\frac{\lambda+2 \mu}{\mu},
\end{gathered}
$$


Solution in the Laplace Transform Domain

Applying the Laplace transform with parameter s defined by the relation

$$
\bar{f}(s)=\int_{0}^{\infty} f(t) e^{-s t} d t,
$$

to both sides of equations (19)-( 23), the following was obtained:

$$
\begin{aligned}
& \frac{1}{r} \frac{\partial}{\partial r}(r \bar{E})=-s \bar{h} \\
& \left.\mid \nabla^{2}-v s-V^{2} s^{2}\right] \bar{h}=s \bar{e} \\
& \left.\nabla^{2} \bar{e}+\varepsilon_{2} v \mid V^{2} s^{2}-\nabla^{2}\right] \bar{h}-\left(1+\tau_{1} s\right) \nabla^{2} \bar{\theta}=s^{2} \bar{e} \\
& \nabla^{2} \bar{\theta}=s\left(1+\tau_{2} s\right) \bar{\theta}+\varepsilon_{1} s\left(1+n \tau_{2} s\right) \bar{e}-\bar{Q} \\
& \bar{\sigma}_{r r}=2 \frac{\partial \bar{u}}{\partial r}+\left(\beta^{2}-2\right) \bar{e}-\beta^{2}\left(1+\tau_{1} s\right) \bar{\theta}
\end{aligned}
$$

Where $\bar{Q}=\frac{R_{a} L_{0}}{\delta_{1} t_{p}^{2}} \frac{e^{h / 2 \delta_{1}}}{\left(s+1 / t_{p}\right)^{2}}$

Eliminating $\bar{e}, \overline{\mathrm{h}}$ from equations (25), (26), and (27), the following was obtained:

$$
\left(\nabla^{6}-a \nabla^{4}+b \nabla^{2}-c\right) \bar{\theta}=-s^{3}\left(V^{2}\left(s+\varepsilon_{2} v\right)+v\right) \bar{Q}
$$

Where

$$
a=s\left[s\left(n \varepsilon_{1} \tau_{2}\left(1+\tau_{1} s\right)+\tau_{2}+V^{2}+1\right)+\varepsilon_{1}\left(1+\tau_{1} s\right)+\right.
$$

$b=s^{2}\left\{s^{2}\left[n \varepsilon_{1} \tau_{2} V^{2}\left(1+\tau_{1} s\right)+\tau_{2}\left(V^{2}+1\right)+V^{2}\right]+\right.$

$s\left[\varepsilon_{1}\left(1+\tau_{1} s\right)\left(n v \tau_{2}+V^{2}\right)\right.$

$\left.\left.+\varepsilon_{2} v\left(\tau_{2}+V^{2}\right)+v\left(\tau_{2}+1\right)+V^{2}+1\right]+\varepsilon_{1} v\left(1+\tau_{1} s\right)+\varepsilon_{2} v+v\right\}$

$c=s^{4}\left(1+\tau_{2} s\right)\left(s V^{2}+\varepsilon_{2} v V^{2}+v\right)$

In a similar manner we can show that $\overline{\mathrm{h}}, \overline{\mathrm{e}}$ satisfy the equations

$$
\begin{aligned}
& \left(\nabla^{6}-a \nabla^{4}+b \nabla^{2}-c\right) \bar{h}=0,(30) \\
& \left(\nabla^{6}-a \nabla^{4}+b \nabla^{2}-c\right) \bar{e}=\mathrm{O}
\end{aligned}
$$

The solutions of equations (29)-(31) bounded for $r$ $=0$ have the forms $\bar{e}=\sum_{i=1}^{3} A_{i} I_{0}\left(k_{i} r\right)$

$\bar{\theta}=\frac{-\bar{Q}}{s\left(1+\tau_{2} s\right)}+\sum_{i=1}^{3} B_{i} I_{0}\left(k_{i} r\right)$,

$\bar{h}=\sum_{i=1}^{3} C_{i} I_{0}\left(k_{i} r\right)$.

where $k_{1}^{2}, k_{2}^{2}$ and $k_{3}^{2}$ are the roots with positive real parts of the characteristic equation:

$k^{6}-a k^{4}+b k^{2}-c=0$,

and $I_{0}$ is the modified Bessel function of the first kind of order zero.

Substituting from equations (33) and (34) into equations (25) and (27), the following was obtained:

$$
\begin{aligned}
& B_{i}=\frac{\varepsilon_{1}\left(s+n \tau_{2} s^{2}\right)}{k_{i}^{2}-\left(s+\tau_{2} s^{2}\right)} A_{i} \quad i=1,2,3 \\
& C_{i}=\frac{s}{k_{i}^{2}-s \delta} A_{i} \quad i=1,2,3
\end{aligned}
$$

where $\delta=v+s V^{2}$.

Substituting from equations (36) and (37) into equations 1 133) and (34), the following was obtained:

$$
\bar{\theta}=\frac{-\bar{Q}}{s\left(1+\tau_{2} s\right)}+\sum_{i=1}^{3} \frac{\varepsilon_{1}\left(s+n \tau_{2} s^{2}\right)}{k_{i}^{2}-\left(s+\tau_{2} s^{2}\right)} A_{i} I_{0}\left(k_{i} r\right),
$$

$$
\bar{h}=\sum_{i=1}^{3} \frac{s}{k_{i}^{2}-s \delta} A_{i} I_{0}\left(k_{i} r\right)
$$

From equations (24) and (39), we obtain

$$
\bar{E}=\sum_{i=1}^{3} \frac{-s^{2}}{k_{i}\left(k_{i}^{2}-s \delta\right)} A_{i} I_{1}\left(k_{i} r\right)
$$

From equations (15) and (32), we get 
$\bar{u}=\sum_{i=1}^{3} \frac{A_{i}}{k_{i}} I_{1}\left(k_{i} r\right)$

(41)

From equations (32), (38), (41) and (28), we obtain

$$
\bar{\sigma}_{r r}=\frac{\beta^{2}\left(1+\tau_{1} s\right) \bar{Q}}{s\left(1+\tau_{2} s\right)}+
$$$$
\sum_{i=1}^{3} A_{i}\left\{\beta^{2}\left(1-\frac{\varepsilon_{1}\left(s+n \tau_{2} s^{2}\right)\left(1+\tau_{1} s\right)}{k_{i}^{2}-\left(s+\tau_{2} s^{2}\right)}\right) I_{0}\left(k_{i} r\right)-\frac{2}{k_{i} r} I_{1}\left(k_{i} r\right)\right\}
$$

The induced fields E0 and h0 in the free space surrounding the cylinder satisfy the following equations:

$$
\frac{\partial \bar{h}_{0}}{\partial r}=-V^{2} s \bar{E}_{0}
$$

$$
\frac{1}{r} \frac{\partial}{\partial r}\left(r \bar{E}_{0}\right)=-s \bar{h}_{0}
$$

Eliminating $\bar{E}_{0}$ between equations (2.43) and (2.44), the following was obtained:

$$
\left\lfloor\nabla^{2}-V^{2} s^{2}\right\rfloor \bar{h}_{0}=0
$$

The solution of equation (2.45) which is bounded at infinity is given by:

$$
\bar{h}_{0}=A_{4}(s) K_{0}(s V r)
$$

where $\mathrm{A}_{4}(\mathrm{~s})$ is some parameter depending on $\mathrm{s}$ only and $\mathrm{K}_{0}$ is the modified Bessel function of the second kind of order zero.

Substituting from equation (46) into equation (43), the following was obtained:

$$
\bar{E}_{0}=\frac{A_{4}(s)}{V} K_{1}(s V r)
$$

The boundary conditions of the problem can be written as:

$$
\begin{aligned}
& \theta(r, t)=0, \quad \sigma_{r r}(r, t)=0 \quad \text { at } \mathrm{r}=\mathrm{a}, \\
& \mathrm{h}=\mathrm{h}_{0} \quad, \quad \mathrm{E}=\mathrm{E}_{0}, \quad \text { at } \mathrm{r}=\mathrm{a}
\end{aligned}
$$

where $\mathrm{a}$ is the reduce of the cylinder. Taking the Laplace transform of both sides of the preceding equations, the following was obtained:

$$
\bar{\theta}(a, s)=0 \text {, }
$$

$$
\begin{aligned}
& \bar{\sigma}_{r r}(a, s)=0 \\
& \bar{h}=\bar{h}_{0} \quad, \bar{E}=\bar{E}_{0} \quad, \quad \text { a } \quad r=a
\end{aligned}
$$

Applying the boundary conditions, the following system of linear equations in the unknown parameters $A_{1}, A_{2}, A_{3}$, and $A_{4}$ could be obtained.

$$
\sum_{i=1}^{3} \frac{\varepsilon_{1}\left(s+n \tau_{2} s^{2}\right)}{k_{i}^{2}-\left(s+\tau_{2} s^{2}\right)} A_{i} I_{0}\left(k_{i} a\right)=\frac{\bar{Q}}{s\left(1+\tau_{2} s\right)}
$$

$\sum_{i=1}^{3} A_{i}\left\{\beta^{2}\left(1-\frac{\varepsilon_{1}\left(s+n \tau_{2} s^{2}\right)\left(1+\tau_{1} s\right)}{k_{i}^{2}-\left(s+\tau_{2} s^{2}\right)}\right) I_{0}\left(k_{i} a\right)-\frac{2}{k_{i} a} I_{1}\left(k_{i} a\right)\right\}$

$=\frac{-\beta^{2}\left(1+\tau_{1} s\right) \bar{Q}}{s\left(1+\tau_{2} s\right)}$

$\sum_{i=1}^{3} \frac{s}{k_{i}^{2}-s \delta} A_{i} I_{0}\left(k_{i} a\right)=A_{4} k_{0}(s V a)$,

(52)

$\sum_{i=1}^{3} \frac{-s^{2}}{k_{i}\left(k_{i}^{2}-s \delta\right)} A_{i} I_{1}\left(k_{i} a\right)=\frac{A_{4}}{V} k_{1}(s V a)$

Solve equations (50)-(53) to find $A_{1}, A_{2}, A_{3}$ and $A_{4}$.

\section{Inversion of the Laplace Transform}

The method used to invert the Laplace transforms in the above equations is outlined as follows: let be the Laplace transform of a function $f(r, t)$, the inversion formula for Laplace transforms can be written as [26]:

$$
f(r, t)=\frac{1}{2 \pi \mathrm{i}} \int_{d-\mathrm{i} \infty}^{d+\mathrm{i} \infty} \mathrm{e}^{\mathrm{st}} \bar{f}(r, s) d s,
$$

where $d$ is an arbitrary real number greater than all the real parts of the singularities of . Taking $s=d+$ i y, the above integral takes the form

$$
f(r, t)=\frac{e^{d t}}{2 \pi} \int_{-\infty}^{\infty} \mathrm{e}^{i t y} \bar{f}(r, d+i y) d y
$$

Expanding the function $h(r, t)=\exp (d t) f(r, t)$ in a Fourier series in the interval $[0,2 \mathrm{~L}]$, we obtain the approximate formula [27]

$$
f(r, t)=f_{\infty}(r, t)+E D,
$$




$$
\begin{aligned}
& f_{\infty}(r, t)=1 / 2 c_{0}+\sum_{k=1}^{\infty} c_{k} \quad, \text { for } 0 \leq t \leq 2 L \\
& c_{k}=\frac{e^{d t}}{L} \operatorname{Re}\left[e^{i k \pi t / L} \bar{f}(d+i k \pi / L)\right]
\end{aligned}
$$

The discrimination error, ED, can be made arbitrarily small by choosing d large enough [27].

As the infinite series in (54) can only be summed up to a finite number $\mathrm{N}$ of terms, the approximate value of $f(r, t)$ becomes

$$
f_{N}(r, t)=1 / 2 c_{0}+\sum_{\mathrm{k}=1}^{\mathrm{N}} c_{k} \quad, \text { for } 0 \leq t \leq 2 L
$$

Using the above formula to evaluate $f(r, t)$ we introduce a truncation error ET that must be added to the discrimination error to produce the total approximation error.

Two methods are used to reduce the total error. First, the `Korrecktur` method is used to reduce the discrimination error. Next, the $\varepsilon$ algorithm is used to reduce the truncation error and therefore to accelerate convergence.

The Korrecktur method uses the following formula to evaluate the function $f(r, t)$

$f(r, t)=f_{\infty}(r, t) \mathrm{e}^{2 d L} f_{\infty}(r, 2 L+t)+E^{\top} D$,

where the discrimination error [27]

Thus, the approximate value of $f(r, t)$ becomes

$f_{N K}(r, t)=f_{N}(r, t) \quad \mathrm{e}^{2 d L} f_{N}(r, 2 L+t)$

$\mathrm{N}^{\prime}$ is an integer such that $\mathrm{N}^{\prime}<\mathrm{N}$.

The $\varepsilon$ algorithm used to accelerate the convergence of the series (54) is described. Let $\mathrm{N}$ be an odd natural number and let

$$
\mathrm{s}_{\mathrm{m}}=\sum_{\mathrm{k}=1}^{\mathrm{m}} \mathrm{c}_{\mathrm{k}}
$$

be the sequence of partial sums of (54). We define the $\varepsilon$ sequence by

$$
\varepsilon_{0, m}=0, \varepsilon_{1, m}=s_{m}, \quad m=1,2,3, \ldots
$$

$$
\begin{aligned}
\varepsilon_{\mathrm{n}+1, \mathrm{~m}} & =\varepsilon_{\mathrm{n}-1, \mathrm{~m}+1}+\frac{1}{\varepsilon_{\mathrm{n}, \mathrm{m}+1}-\varepsilon_{\mathrm{n}, \mathrm{m}}}, \\
\mathrm{n}, \mathrm{m} & =1,2,3, \ldots
\end{aligned}
$$

It can be shown that [27] the sequence

$\varepsilon_{11}, \varepsilon_{31}, \ldots \ldots \ldots \ldots \ldots . . ., \varepsilon_{N 1, \ldots \ldots}$.

Converges to $f(r, t)+E D-C_{0} / 2$ faster than the sequence of partial sums

$$
\mathrm{sm} \quad, \quad \mathrm{m}=1,2,3, \ldots .
$$

The actual procedure used to invert the Laplace Transforms consists of using equation (57) together with the $\varepsilon$-algorithm. The values of $d$ and $\mathrm{L}$ are chosen according the criteria outlined in [27].

\section{Numerical results and discussion}

We shall apply our results to the copper material. The material properties are

$$
\begin{aligned}
& \lambda=7.76 \times 10^{10} \mathrm{~kg} \mathrm{~m}^{-1} \mathrm{~s}^{-2}, \mu=3.86 \times 10^{10} \mathrm{~kg} \mathrm{~m}^{-1} \mathrm{~s}^{-2} \\
& , \rho=8954 \mathrm{~kg} \mathrm{~m}^{-3}, \\
& k=386 \mathrm{~kg} \mathrm{~m} \mathrm{~K}^{-1} \mathrm{~s}^{-3}, C_{E}=381 \mathrm{~m}^{2} \mathrm{~K}^{-1} \mathrm{~s}^{-2}, \\
& T_{0}=293 \mathrm{~K}, \alpha_{t}=1.78 \times 10^{-5} \mathrm{~K}^{-1}, \\
& \mu_{0}=4 \pi \times 10^{-7} \mathrm{H} \mathrm{m}^{-1}, \tau_{2}=0.02, \\
& \varepsilon_{0}=10^{-9} / 36 \pi \mathrm{Fm}^{-1}, L_{0}=1 \times 10^{11} \mathrm{Jm}^{-2}, \\
& R_{a}=0.5, t_{p}=2.0, h=0.1, \delta=0.01, \tau_{1}=0.01
\end{aligned}
$$

All field quantities: temperature, displacement, stress, induced magnetic field and induced electric field are dependent only on $t, r$. The problem was solved for one value of time namely $t=0.1$ The graphs for the temperature, displacement, stress, induced magnetic field and induced electric field are shown in Figs. (2-6), respectively. Dotted lines represent the solution for coupled thermoelasticity (CTE), dashed lines represent the solution for Green-Lindsay (G-L) model and solid lines represent the case Lord-Shulman (L-S) model.

In the coupled thermoelasticity (CTE) we put $\tau_{1}=\tau_{2}=0, n=1$, in Green-Lindsay (G-L) model put $\tau_{1}=0.01, \tau_{2}=0.02, n=0$, in Lord-Shulman (L-S) model put $\tau_{1}=0, \tau_{2}=0.02, n=1$. In Fig. (2) the temperature distribution, it was observed that the curves have the same behavior for the three theories of thermoelasticity. There are 
starting from out surface of the cylinder $r=\mathrm{a}$, the heat is increasing until it constant.

In Figs. (3-4) displacement distribution and stress, we observe all the curves start with negative values of z-axes, then rapidly increase to a maximal positive value and there after continuously decrease to zero value. In Fig. (5) the induced magnetic filed is the direction of the negative value of $\mathrm{z}$-axes, the value of induced magnetic field is change between inside and out side of the cylinder. In Fig. (6) the value of induced electric filed is zero at $r=0$, and it will increase inside and outside the cylinder until it becomes zero.

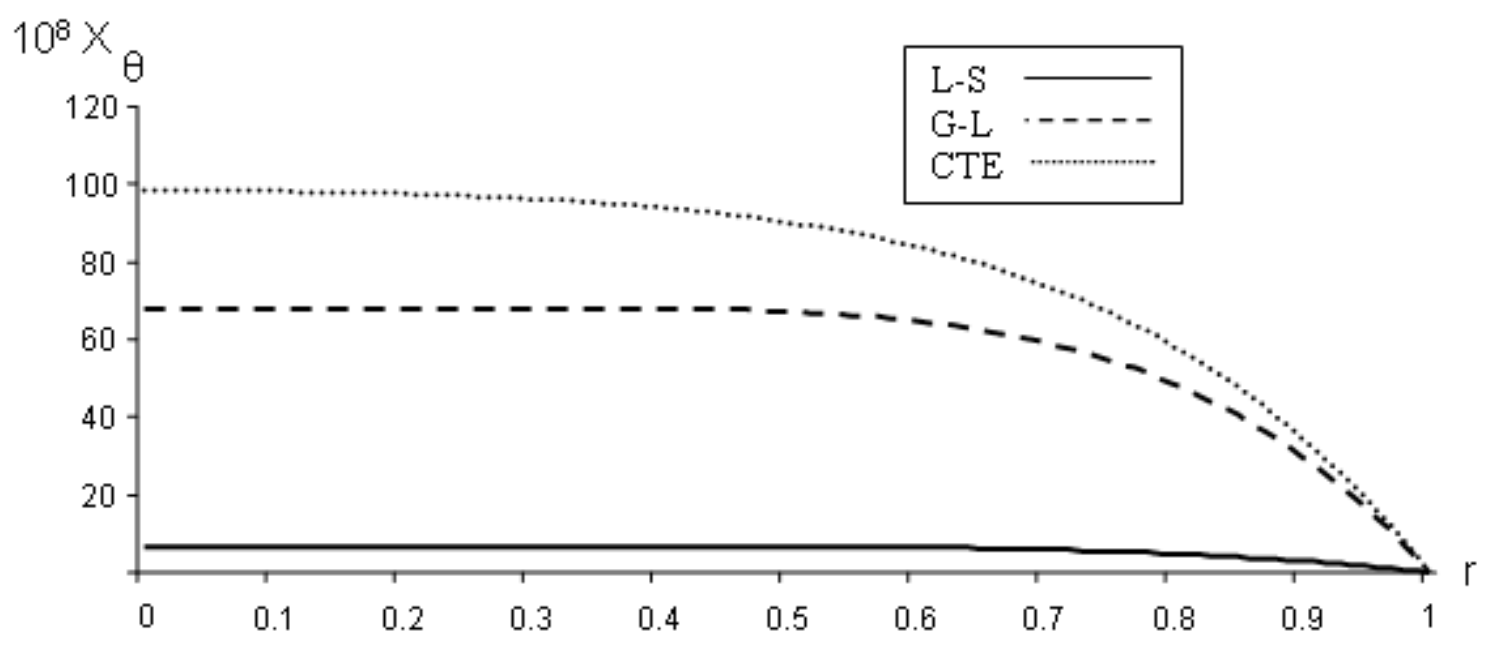

Figure (2) Temperature distribution

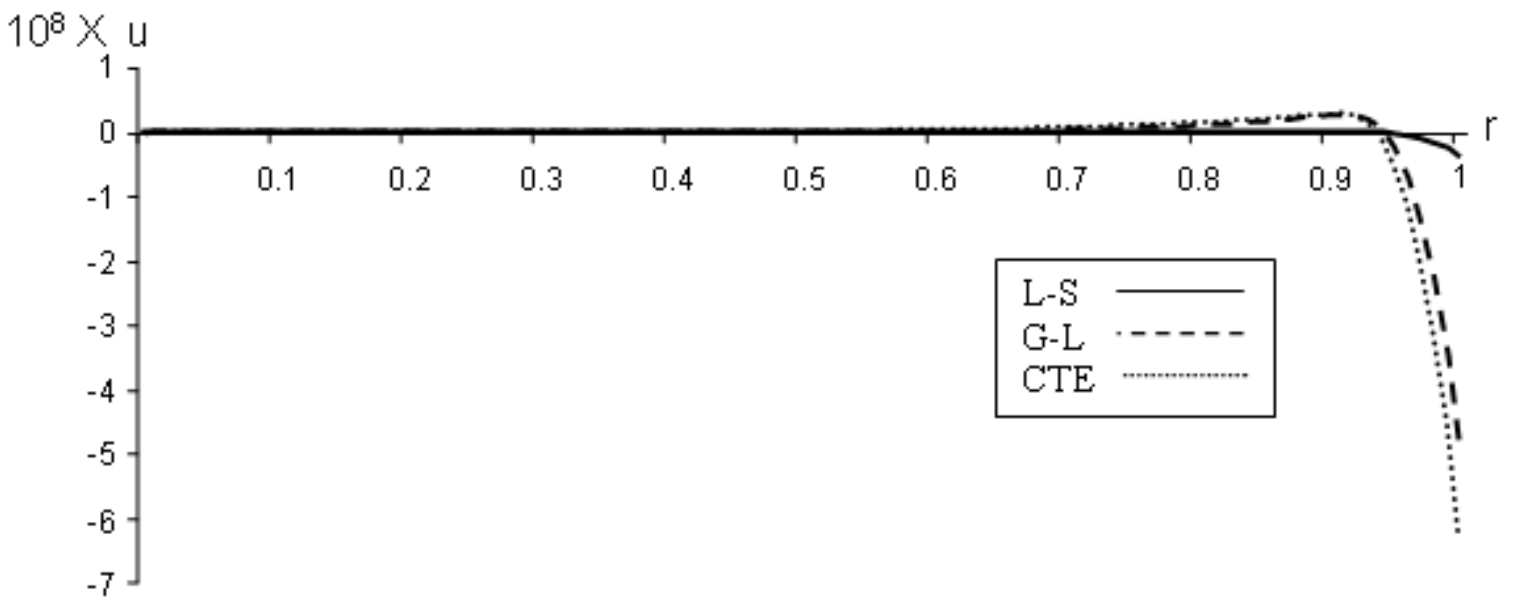

Figure (3) Displacement distribution 


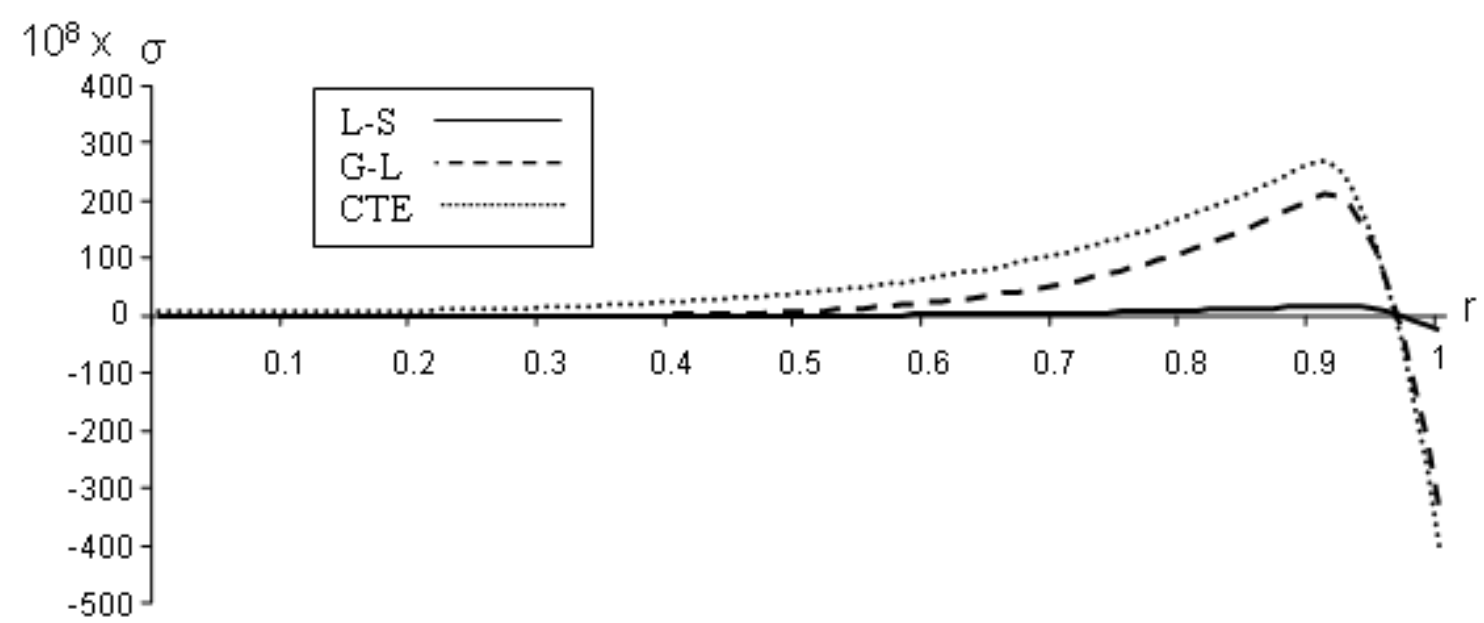

Figure (4) Stresses distribution

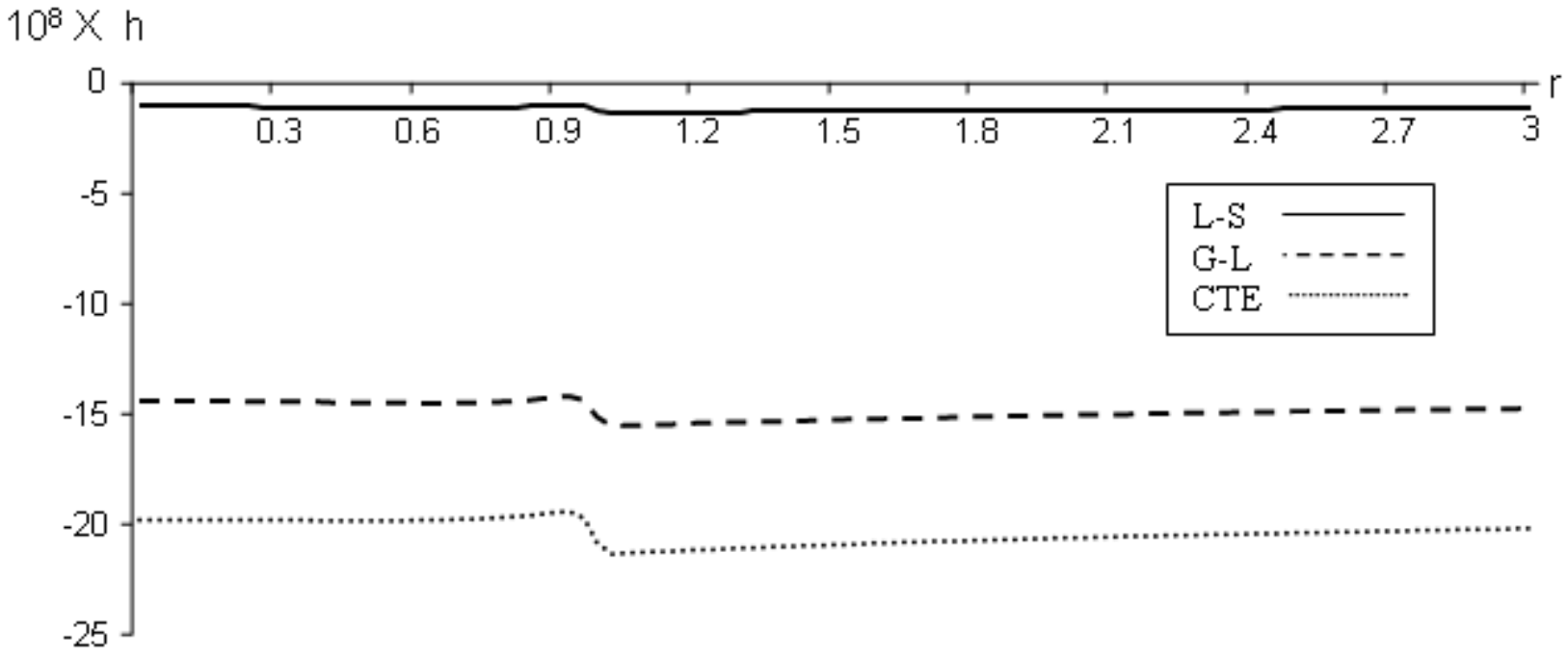

Figure (5) Induced magnetic field

$10^{8} \times \mathrm{E}$

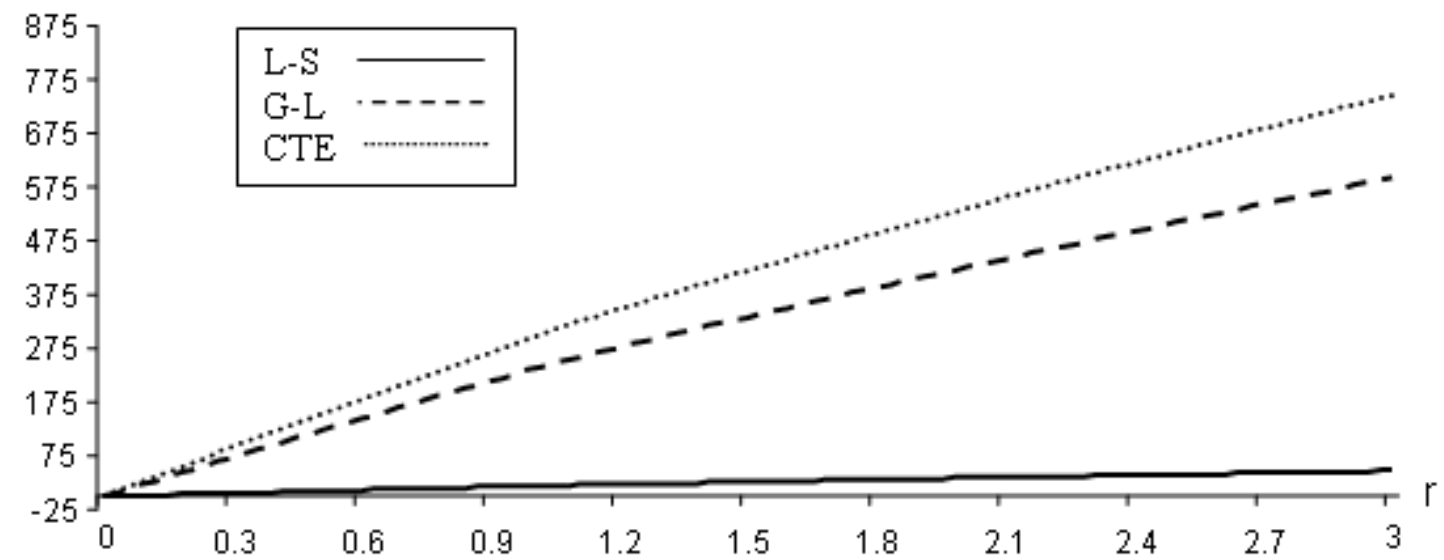

Figure (6) Induced electric field 


\section{References}

1- Lord H, Shulman Y.A. generalized dynamical theory of thermoelasticity. J. Mech Phys Solid 15: 299-309. (1967)

2- Hany Sherief, Nasser M. El-Maghraby. An Internal Penny-Shaped Crack in an Infinite Thermoelastic Solid". J. Thermal Stresses 26: 333-352 (2003)

3- Hany H. Sherief, Nasser M. El-Maghraby. A Mode-I Crack Problem for an Infinite Space in Generalized Thermoelasticity. J. Thermal Stresses 28:465-484 (2005).

4- Hany Sherief, Farid Hamza. Generalized TwoDimensional Thermoelastic Problems in Spherical Regions under Axisymmetric Distributions. J Thermal Stresses 19: 55-76 (1996)

5- Hany Sherief, Farid Hamza. Generalized Thermoelastic Problem of a Thick Plate Under Axisymmetric Temperature Distribution. J Thermal Stresses 17: 435-452 (1994)

6- Hany Sherief, Magdy A Ezzat. Solution of the Generalized Problem of Thermoelasticity in the form of Series of Functions. J Thermal Stresses 17: 75-95 (1994)

7- Hany Sherief, Ranjit Dhaliwal. A Generalized One-Dimensional Thermal Shock Problem for Small Times. J Thermal Stresses 4: 407-420 (1981)

8- Hany H Sherief, Farid A Hamza, Amany M ElSayed. Theory of Generalized Micropolar Thermoelasticity and an Axisymmetric HalfSpace Problem. J Thermal Stresses 28: 409-437 (2005)

9- Hany H Sherief, Mohammed N Allam, Mohammed A El-Hagary. Generalized Theory of Thermoviscoelasticity and a Half-Space Problem. Int J Thermophys 32:1271-1295 (2011)

10- Y. Sun, D. Fang, M. Saka , A. K. Soh, LaserInduced Vibrations of Micro-Beams under Different Boundary Con- ditions, International Journal of Solids and Structures 45:1993-2013 (2008)

11- F. A. McDonald. On the Precursor in LaserGenerated Ultrasound Waveforms in Metals. Applied Physics Let-ters.56: 230-232 (1990)

12- A.M. Abd El-Latief, S. E. Khader. Fractional model of thermoelasticity for a half-space overlaid by a thick layer Z. Angew. Math. Mech. 95: 511-518 (2015)
13- Nayfeh A, Nemat-Nasser S Electromagnetothermoelastic plane waves in solids with thermal relaxation. J Appl Mech Series E 39:108 113 (1972)

14- Hany H. Sherief - S.E. Khader. Propagation of discontinuities in electromagneto generalized thermoelasticity in cylindrical regions Meccanica 48:2511-2523 (2013)

15- A. E. Green and K. A. Lindsay. Thermoelasticity, J. Elasticity, 2: 1-7 (1972)

16- H. Sherief. State Space Approach to Thermoelasticity with Two Relaxation Times. Int. J. Eng. Sci., 31: 1177-1189 (1993)

17- H. Sherief, Problem in Electromagneto Thermoelasticity for an Infinitely Long Solid Conducting Circular Cylinder with Thermal Relaxation. Int. J. Eng. Sci., 32: 435-452 (1994)

18- A. E. Green and P. M. Naghdi, A re-examination of the basic postulate of thermo-mechanics. Proc. Roy. Soc. London, 432: 171-194 (1991)

19- A. E. Green and P. M. Naghdi, (1992) An unbounded heat wave in an elastic solid. J. Thermal Stresses 15:253-264.

20- A. E. Green, P. M. Naghdi (1993) Thermoelasticity without energy dissipation". J. Elasticity, 31: 189-208

21- D. S. Chandrasekharaiah (1996) A uniqueness theorem in the theory of thermoelasticity without energy dissipation, J. Thermal Stresses, 19: 267-272.

22- D. S. Chandrasekharaiah ( 1996) A note on the uniqueness of solution in the linear theory of thermoelasticity without energy dissipation. J. Elasticity, 43: 279-283.

23- D. S. Chandrasekharaiah (1996) Onedimensional wave propagation in the linear theory of thermoelasticity without energy dissipation. J. Thermal Stresses, 19: 695-710.

24- D. S. Chandrasekharaiah, (1996) Thermoelastie plane waves without energy dissipation. Mech. Res. Comm, 23: 549-555.

25- A. M. Abd El-Latief , S. E. Khader (2014) Exact Solution of Thermoelastic Problem for a OneDimensional Bar without Energy Dissipation" ISRN Mechanical Engineering, Volume 2014, Article ID 694259, 6 pages

26- Churchill R V (1972) Operational Mathematics, Third edition, McGraw-Hill Book Company, New York.

27- Honig G, Hirdes U (1984) A Method For The Numerical Inversion of the Laplace Transform. J Comp Appl Math 10:113-132. 